\title{
Effects of spacing on same-different judgments to simple outline forms
}

\author{
MURRAY J. WHITE and DIANNE E. GREEN \\ Victoria University of Wellington, Wellington, New Zealand
}

\begin{abstract}
Twenty-four subjects made same-different responses to pairs of simple outline forms. The forms were either physically identical or numerically identical (having the same number of composing elements) or physically and numerically different. The forms were shown in either spatially adjacent or spatially separated positions. In two independent conditions, same responses for physically identical forms were significantly faster when the forms were shown spatially adjacent than when they were shown spatially separated. Same responses for numerically identical forms were not affected by spacing. The results were seen as supporting Posner's (1969) interpretation of spacing effects observed in Hochberg's (1968) experiments, in terms of matching stimuli on the basis of physical equivalence, and on the basis of nominal equivalence properties.
\end{abstract}

Using a simple visual comparison task, Hochberg (1968) had subjects judge whether two vertically printed words were the same or different. The dependent variable was the number of glances needed to make the correct judgment. Hochberg manipulated stimulus familiarity by reversing the letters in some word pairs and jumbling the letter sequences in others. When the words were presented in spatially adjacent positions, the comparison time and the number of glances required to make a same-different judgment were the same for familiar and for unfamiliar stimuli. When the words were separated by 20 deg visual angle, unfamiliar stimuli took significantly longer times to match than did familiar stimuli. Hochberg argued that in the indirect comparison (20-deg spacing) condition, subjects had to base matches on the remembered properties of stimuli. The more efficiently remembered familiar stimuli were matched faster than the less efficiently remembered unfamiliar stimuli.

Posner (1969) interpreted Hochberg's results in terms of time differences in matching stimuli on the basis of physical equivalence and on the basis of nominal equivalence properties. He suggested that Hochberg's results could be explained by assuming that subjects were matching closely spaced words on the basis of physical equivalence information and matching widely spaced words on the basis of name equivalence information. Posner has elsewhere suggested that name matching, unlike physical stimulus matching, requires a comparison with information retrieved from short-term memory (Posner, Boies, Eichelman, \& Taylor, 1969; Posner \& Mitchell, 1967).

The present experiment was addressed to Posner's explanation of the spacing effect observed in Hochberg's experiments. Stimuli consisted of pairs of outline forms

This research was supported by Grant $95 / 75$ from Victoria University of Wellington. The authors are grateful to Dr. Geoff White for helpful advice. which could be matched on the basis of physical identity and numerical identity, or numerical identity alone. It was reasoned that matching stimuli on the basis of numerical identity would require a higher level of information processing than would matching stimuli on the basis of physical equivalence. Like Posner's name-matching (Node 2) task, numerical identity matching would require a comparison of nominally encoded information; in this case, the number of elements making up each stimulus.

Two basic forms served as stimulus forms, one composed of four, and one of five, open circles. Each of these forms was also shown in a position rotated $45 \mathrm{deg}$ from the normal. The forms were regular, symmetrical, and simple, and were considered, a priori, to be equally familiar.

The experiment tested the following hypotheses: (1) For physically identical and numerically identical (PI) forms, same responses based on the physical equivalence of the forms can be made when the forms are spatially adjacent. When the forms are spatially separated, same responses must be based on a comparison of nonsensory (memorial) equivalence properties. (2) For numerically identical and physically different (NI) forms, same responses must be based on a comparison of nonsensory equivalence properties. Such matches will not be affected by spacing.

The predictions were: (a) Same response times (RTs) for PI matches will be faster when the forms are spatially adjacent than when they are spatially separated. (b) Same RTs for NI matches will not be affected by the degree of spatial separation. These predictions were tested in two independent experimental conditions, similar to those used by Posner and Mitchell (1967, Experiments 1 and 2).

\section{METHOD}

Stimuli and Apparatus

Each of 24 cards showed two outline forms, mounted on the 
Figure 1

Stimulus Pairs

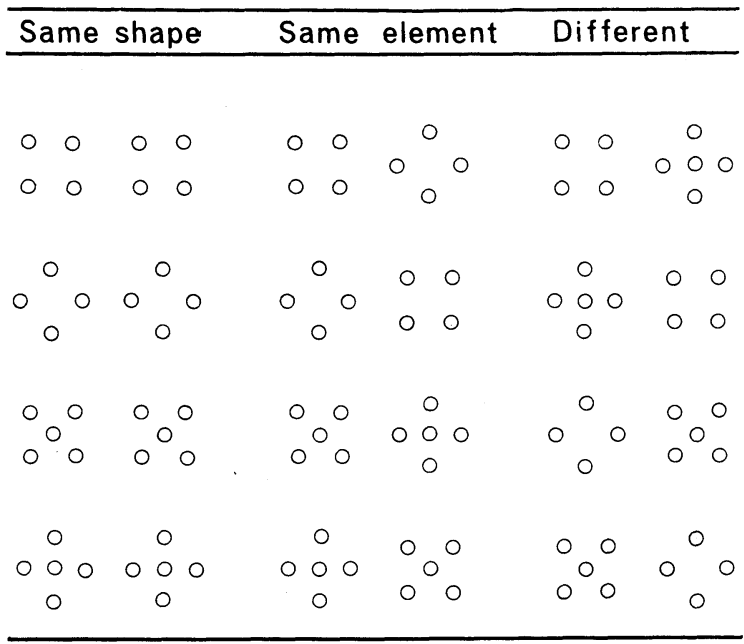

horizontal midline, one to the left and one to the right of fixation. Four simple forms were used as stimuli. These were represented by a pattern of either four or five open circles. A single form subtended visual angles of $1.4 \times 1.4 \mathrm{deg}$ or $1.8 \mathrm{deg}$, and a single circle (element) an angle of .4 deg. On each of eight cards, the two forms had the same shape and the same numbers of elements (PI forms). On each of another eight cards, the two forms had different shapes and the same numbers of elements (NI forms). On each of another eight cards, the two forms had different shapes and different numbers of elements (D forms). Within each of these three sets of eight cards, four pairs of forms had 2.9 deg visual angle separation between centers and four pairs had 6.7 deg visual angle separation between centers. The forms are shown in Figure 1.

Stimuli were presented in a Gerbrands tachistoscope. A fixation field was initially illuminated for $2 \mathrm{sec}$, and this was followed by a stimulus exposure of $1 \mathrm{sec}$. Following offset of stimulus exposure, an illuminated field was shown for $2 \mathrm{sec}$. The luminance of all three fields was matched at $17 \mathrm{~cd} / \mathrm{m}^{2}$, and the intertrial interval was dark. Subjects responded by pressing one of two telegraph keys with the right or left hand. The keys were coupled to the tachistoscope starter and a Nixie-tube display, and RTs accurate to $1 \mathrm{msec}$ were available.

\section{Procedure}

In one condition (pure PI), the subject was instructed to respond same whenever two forms having the same shape were shown, and different whenever two forms having different shapes were shown. A session consisted of 64 trials in which the eight PI and eight D cards were presented in two different random orders at two orientations $(0 \mathrm{deg}$ and $180 \mathrm{deg})$ to each subject.

In a second condition (pure NI), the subject was instructed to respond same whenever two forms having the same numbers of elements were shown, and different whenever two forms having different numbers of elements were shown. A session consisted of 64 trials in which the eight NI and the eight D cards were shown, in different random orders to each subject.

In a third condition (mixed PI and NI), the instructions were the same as for the pure NI condition. A session consisted of 64 trials made up of two random orders of the eight PI cards, the eight NI cards, and the eight D cards (each D card being represented twice). Subjects in each condition were familiarized with the respective stimuli and tasks prior to each experimental run.

Eight subjects served in each of the three conditions. A subject was assigned randomly to a particular condition in the order he/she volunteered for the experiment. The subject was instructed to respond by rapidly pressing one of the two telegraph keys with the left or right hand, depending on whether the stimuli on each trial were the same or different. Keys (1 and 2), hands (left and right), and responses (same and different) were counterbalanced in each group of eight subjects. Subjects were given feedback only about the accuracy of their responses.

\section{Subjects}

Eleven female and 13 male introductory psychology students volunteered for the experiment. Each subject was screened to insure normal or corrected-to-normal vision. Each subject was paid $\$ 1$ for participating in the experiment. (The results of one subject assigned to the pure PI condition were discarded because of a power failure during testing. The results of one subject assigned to the mixed condition were also discarded because of an inability to respond faster than $1 \mathrm{sec}$. Two other subjects were recruited from the same pool, and the results for these new subjects were included in the analyses.)

\section{RESULTS AND DISCUSSION}

Mean RTs for same and different responses in pure and mixed conditions are shown in Table 1 . Mean errors for the pure PI, pure NI, and mixed conditions were $1 \%, 4.3 \%$, and $5.7 \%$, respectively. No analysis was made of these few errors, and error RTs were not included in the data analyses.

\section{Same Responses: Pure Condition}

Spatially adjacent forms were matched significantly faster than spatially separated forms $[802 \mathrm{msec}$ and $841 \mathrm{msec}$, respectively, $F(1,14)=5.90, \mathrm{p}<.05]$. Matching on the basis of physical identity $(796 \mathrm{msec})$ was not faster than matching on the basis of numerical identity ( $848 \mathrm{msec}), \mathrm{F}<1$. The absence of a significant matching effect could be attributed to subject variability since, in the pure conditions, matching was a betweensubjects factor. The Spacing by Matching interaction was significant $[F(1,14)=5.30, p<.05]$. Individual comparison tests showed that for PI matches spatially adjacent forms were matched faster than spatially separated forms $[t(7)=3.78, p<.01]$. For NI matches there was no spacing effect $(t<1)$.

\section{Same Responses: Mixed Condition}

Spatially adjacent forms were not matched faster than spatially separated forms $[779 \mathrm{msec}$ and $839 \mathrm{msec}$, respectively, $F(1,7)=3.27, p>.05]$. Matching on the basis of physical identity was faster than matching on the basis of numerical identity [ $775 \mathrm{msec}$ and $843 \mathrm{msec}$, respectively, $F(1,7)=29.56, \mathrm{p}<.01]$. The Spacing by Matching interaction was again significant $[F(1,7)=$ $7.24, \mathrm{p}<.05]$. Individual comparisons showed that for PI matches, spatially adjacent forms were matched faster than spatially separated forms $[t(7)=3.56, p<.01]$, while for NI matches there was no spacing effect $(t<1)$.

\section{Different Responses}

Spacing was the only significant effect $[\mathrm{F}(1,21)=$ $28.39, \mathrm{p}<.01]$. 
In both the pure and mixed conditions, the Spacing by Matching interactions were significant and the form of these interactions was exactly that predicted. Same PI matches were faster for spatially adjacent forms and same NI matches were not affected by spacing. These results are consistent with the view that subjects were able to base matches on the physical equivalence of forms when the forms were in close proximity, and that matches were based on a comparison of nonsensory equivalence properties when forms were spatially separated. It is of interest to note that the present results were observed with a stimulus spacing difference of only $3.8 \mathrm{deg}$, that is, $6.7-2.9 \mathrm{deg}$ (cf. Hochberg, 1968).

Table 1

Mean Response Times (Milliseconds) for Physically Identical (PI), Numerically Identical (NI), and Different (D) Matches in Pure and Mixed Conditions

\begin{tabular}{lllllr}
\hline & & & \multicolumn{3}{c}{ Spacing (Degrees) } \\
\cline { 4 - 6 } Condition & Response & Match & 6.7 & 2.9 & $6.7-2.9$ \\
\hline Pure & Same & PI & 834 & 758 & 76 \\
Pure & Same & NI & 849 & 847 & 2 \\
Mixed & Same & PI & 792 & 714 & 78 \\
Mixed & Same & NI & 868 & 849 & 19 \\
Pure & Different & (PI) & 803 & 747 & 56 \\
Pure & Different & (NI) & 875 & 811 & 64 \\
Mixed & Different & & 873 & 795 & 78 \\
\hline
\end{tabular}

The significant effect of spacing on different RTs in the pure and mixed conditions, and the similarity between the different RTs and the same NI RTs (see Table 1), suggest that different matches for spatially adjacent forms were based on a comparison of shape identity/difference, and different matches for spatially separated forms were based on a comparison of element identity/difference information.

The present results thus agree with Posner's interpretation of Hochberg's results. The faster PI matches for spatially adjacent forms can be interpreted as reflecting a rapid stimulus comparison based on physical equivalence (perceptual) properties. The slower PI matches for spatially separated forms can be seen as reflecting a slower comparison based on element equivalence (memorial) properties.

\section{REFERENCES}

HochberG, J. E. In the mind's eye. In R. N. Haber (Ed.), Contemporary theory and research in visual perception. New York: Holt, Rinehart, \& Winston, 1968.

PosNer, M. I. Abstraction and the process of recognition. In J. T. Spence \& G. Bower (Eds.), The psychology of learning and motivation (Vol. 3). New York: Academic Press, 1969.

Posner, M. I., Boies, S. J., Eichelman, W. H., \& Taylor, R. L. Retention of visual and name codes of single letters. Journal of Experimental Psychology, 1969, 79, No. 1, Part 2.

Posner, M. I., \& Mitchell, R. F. Chronometric analysis of classification. Psychological Review, 1967, 74, 392-409.

(Received for publication August 12, 1976.) 